\title{
Design of 3D Lidar Railway Obstacle Monitoring System
}

\author{
Rui Dou ${ }^{1,}$, Yuting Zhang ${ }^{1, ~ a, ~ Y u n r u i ~ B a i ~}{ }^{2, b}$, Xuechun Lin ${ }^{2, b}$ \\ ${ }^{1}$ China University of Petroleum, Beijing 102249, China \\ ${ }^{2}$ University of Chinese Academy of Sciences, Beijing 100083, China \\ a2018215508@student.cup.edu.cn, bbaiyunrui@semi.ac.cn
}

Keywords: Foreign body intrusion, monitoring system, data acquisition, point cloud.

\begin{abstract}
With the rapid development of our country's railway construction, the demand for railway foreign body intrusion monitoring is growing. Based on the monitoring requirements of strong anti-interference ability, high resolution, and complete monitoring information, this paper introduces the basic principles of laser radar obstacle monitoring, and proposes an overall scheme for railway obstacle monitoring system based on laser radar. The motion control, data acquisition technology and point cloud conversion algorithm for depth data are introduced in detail. Simulation experiments show that the point cloud imaging is clear, which meets the actual requirements of foreign body intrusion monitoring.
\end{abstract}

\section{Introduction}

Our country's terrain structure is relatively complex, and railways often have to travel across mountains, which puts forward higher requirements for the safe operation of railways. Railway disasters in our country are mainly occurred on the roadbed [1], and the entry of foreign body is very easy to cause derailment, overturning and other dangers. Therefore, the research of railway foreign body intrusion monitoring is of great significance.

Railway obstacle monitoring is mainly divided into two categories: contact monitoring and non-contact monitoring. Contact monitoring detects falling rocks through sensors installed on the protective net [2], there are certain missing and false alarms, and it is unable to identify the shape and size of foreign body falling into the rail, unable to assess the risk level. In contrast, non-contact monitoring has low energy consumption, low failure rate, convenient installation and debugging, and has complete monitoring information, which has rich theoretical basis and broad development prospects [3]. In this paper, a high-precision lidar monitoring system is designed and implemented by using non-contact detection means and a combination of various technologies. The system can monitor the rail and its surrounding area within 1 meter in real time, greatly reducing the impact on the recognition accuracy in the unconventional weather conditions such as rain, snow, fog and haze. Once the foreign body invasion occurs, timely and accurate alarm and alarm content will be sent to the control center to ensure the safe operation of the train.

\section{System principle and scheme}

\subsection{Principle of lidar obstacle monitoring.}

Lidar uses a laser with extremely small divergence as the signal source. The transmitting system sends an optical pulse signal [4]. The pulse signal spreads to the target object and causes scattering. Part of the reflected pulse is collected by the receiving system. By accurately measuring the flight time from the emission to the reflected pulse signal being received by the receiving system [5]. The transmission distance can be obtained.

$$
d=c t_{0} / 2
$$

Where: $d$ is the target distance. Because the light pulse signal propagates at the speed of light. $\mathrm{c}$ is the speed of light; $t 0$ is the flight time of the laser pulse. the scanning range of the three-dimensional 
lidar is shown in Figure 1. The laser light emitted by the laser is shaped and expanded by the emission scanning system to the target Directional emission to achieve scanning of a plane perpendicular to the radar axis centered on the light source. In the horizontal direction, a 50rad/s motor drives the light source to rotate to achieve beam expansion. In the traditional way, only when the quality of the optical pulse is ensured, the size and the arrangement interval of the laser are reduced as far as possible, and the vertical resolution can be improved accordingly. Therefore, this design is based on a high-resolution two-dimensional lidar, using full-closed-loop pitch motion control to drive the lidar to high-precision uniform angular velocity pitch movement within the monitoring range, and realize real-time scanning with a vertical resolution of $0.1^{\circ}$.

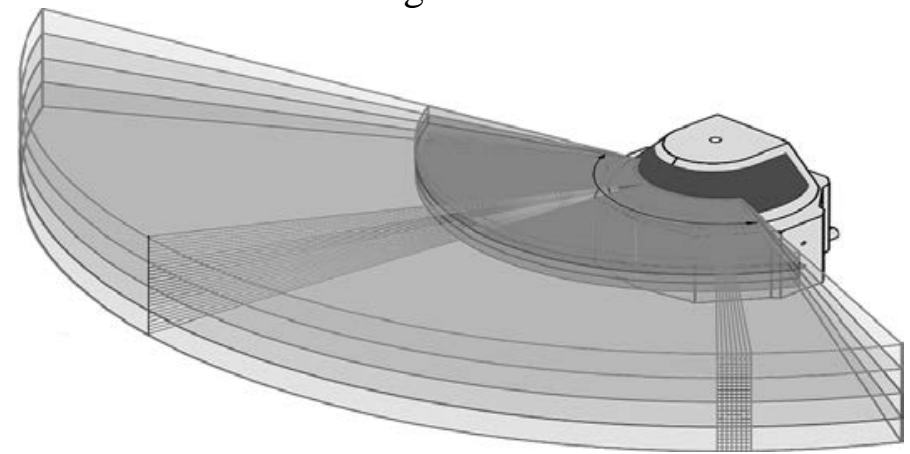

Fig. 1 Schematic diagram of lidar scanning

After obtaining the depth information of the target relative to the lidar, combined with the position of the lidar and the light pulse emission direction and pitch angle information, a three-dimensional coordinate system is established with the lidar as the origin, and the coordinates $\mathrm{X}, \mathrm{Y}, \mathrm{Z}$ of each target point can be accurately calculated. The emission frequency of the light pulse is $25.5 \mathrm{kHz}$, full coverage of the monitoring range is continuously scanned, the depth data of all target points are collected, and then the depth data point cloud is converted and imaged to obtain an accurate three-dimensional image.

\subsection{System overall scheme design.}

The three-dimensional lidar railway obstacle monitoring system is composed of mechanical pitching scanning system, data acquisition and transmission system and data processing system. mechanical pitching scanning system mainly includes mechanical pitching device, servo motor and control circuit; data acquisition and transmission system is composed of lidar, 422 serial port, 485 serial port and Ethernet interface; data processing system is provided with central processing unit with ARM core processor AM335X as the core. The overall design block diagram of the system is shown in Figure 2.

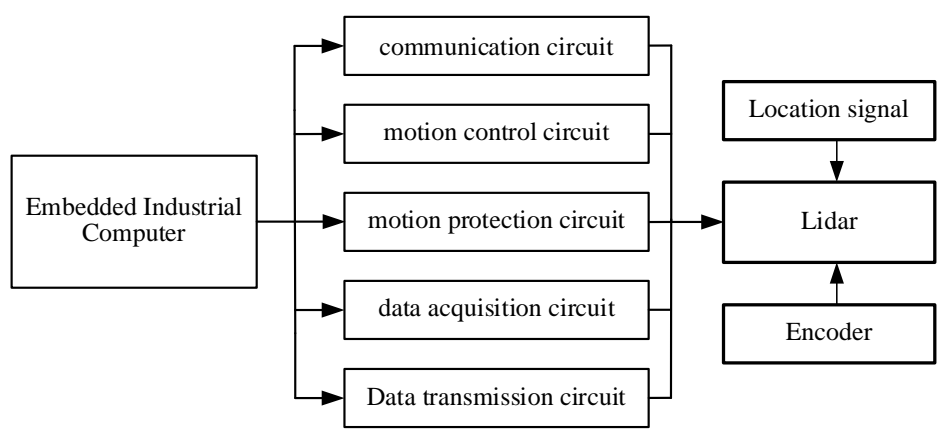

Fig. 2 System overall structure diagram

\section{Hardware design}

\subsection{Mechanical pitching scanning system.}

Considering the accuracy requirement of the monitoring system for scanning, the servo motor is selected to drive the radar to reciprocate and pitch within the monitoring range [6]. The embedded industrial computer, control circuit, servo driver, servo motor, encoder and position feedback 
structure on the radar together form a fully closed-loop control system. The industrial computer is the center of the fully closed-loop control system, According to the control circuit and the status information returned by the radar, the industrial control computer plans the system operation as a whole [7], and cooperates with the protection module to ensure the safe and stable operation of the system.

\subsection{Data acquisition and transmission system.}

The synchronous data acquisition is completed by the cooperation of communication circuit, motion control circuit, motion protection circuit and data acquisition circuit. The communication circuit uses 422 serial port to realize full duplex point-to-point communication between the motion control circuit and the embedded industrial computer, and real-time transmission of motion control protocol and instructions. The motion control circuit controls the corresponding equipment to complete the command action according to the protocol and instruction [8]. When the system runs abnormally, the motion protection circuit responds in time, and uses 485 serial port to transmit the fault information to the industrial control host.

The data acquisition circuit realizes data communication between embedded industrial control host and radar through Ethernet [7]. When the embedded industrial control host sends the motion command, it also issues the data acquisition command, and calculates the acceleration time of the servo motor, so as to ensure that the motor starts to run at a constant speed and start data synchronous acquisition. Using multithreading operation mode, at the same time of data collection, the collected depth data is converted to point cloud [9] and stored, and then sent to the server through Ethernet for foreign body intrusion identification processing.

\section{Data processing}

According to the actual application requirements, the mathematical model is established as shown in the figure 3.

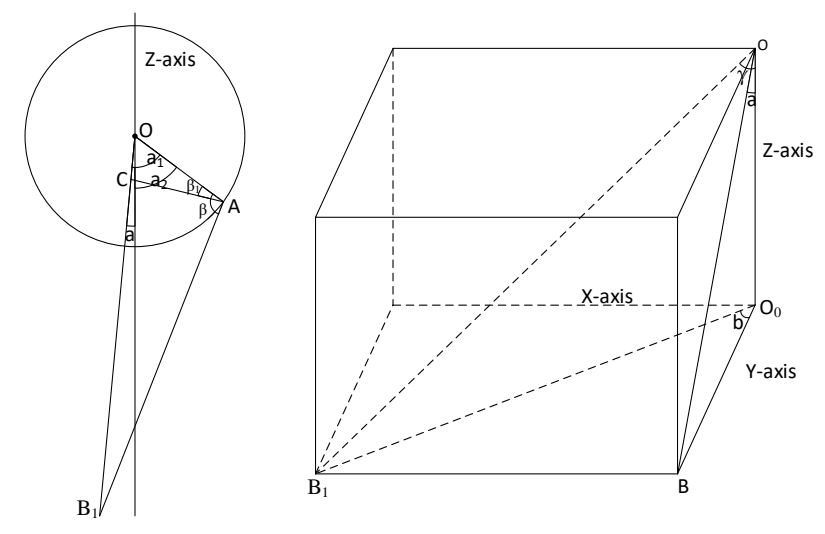

Fig. 3 Range of motion model

Among them, The left is the side view and the right is the front view.

In order to ensure the scanning accuracy, the center of the pitch motion is placed on the radar center of gravity $\mathrm{O}$ point, where $\mathrm{A}$ is the pulse emission point, $\mathrm{B}_{1}$ is the target point, $\mathrm{AB}_{1}$ is the distance between the light source and the target point, and the coordinate system is established with the $\mathrm{O}$ point as the coordinate origin. The point depth $\mathrm{OB}_{1}$ is:

$$
\left|O B_{1}\right|=\sqrt{|O A|^{2}+\left|A B_{1}\right|^{2}-2|O A||A B| \cos \beta_{1}}
$$

When the angle between $\mathrm{OB}_{1}$ and $\mathrm{x}$-axis is $\alpha$, and the angle between $\mathrm{OB}_{1}$ and $\mathrm{y}$-axis is $\mathrm{b}$, and linecnt is the number of radar scanning lines, and $\gamma$ is the angle range then

$$
\alpha_{1}=\arctan \frac{\left|A B_{1}\right|^{2}+\left|O B_{1}\right|^{2}}{2 *\left|A B_{1}\right| *\left|O B_{1}\right|}
$$




$$
\begin{aligned}
& \alpha=\gamma_{1}-\frac{\left(\gamma_{2}-\gamma_{1}\right) * \mathrm{i}}{\text { linecnt }}-\alpha_{1} \\
& b=0.167 *(j-510)
\end{aligned}
$$

Then, in the coordinate system with point $\mathrm{O}$ as the coordinate origin, the coordinates of the target point are:

$$
\begin{aligned}
& x=\operatorname{deph} * \sin b \\
& y=\operatorname{deph} * \sin \alpha \cos b \\
& z=\operatorname{deph} * \cos \alpha \cos b
\end{aligned}
$$

\section{Experiment and analysis}

Test the performance of the obstacle monitoring system developed. The test is divided into two stages, performance test and stability test.

The road was selected as the scanning object, bicycles, tool kits and stones (the stone size was 3.2 $* 1.7 * 3.5 \mathrm{~cm})$ as the monitoring objects. The 3-D point cloud Figure of 3D lidar railway obstacle monitoring system is shown in the figure 4 . The visual observation of the monitored object is clear and visible, and the foreign body on the road surface, road shoulder and road surface can be easily distinguished.

Then, the obstacle monitoring system was tested continuously for one month. The detection performance was not affected by low temperature and moderate snow weather, and it was still stable under $85^{\circ} \mathrm{C}$ high temperature without stray interference. The point cloud shows that the system fully meets the requirements of foreign body intrusion identification.

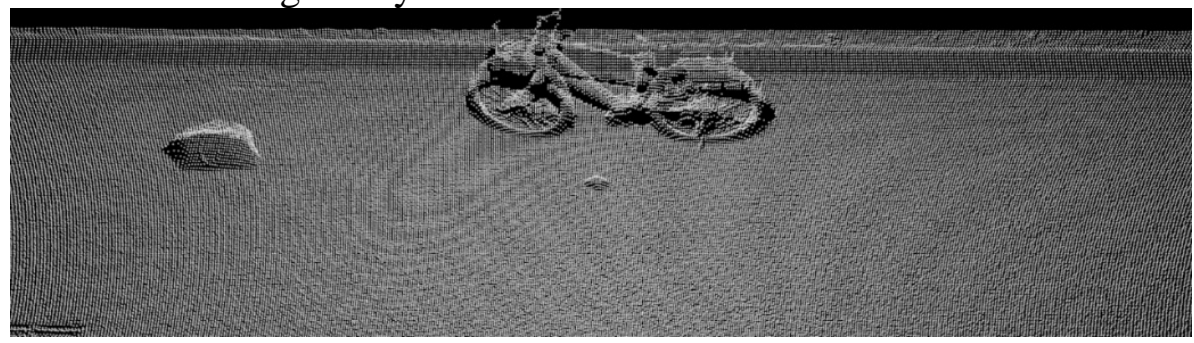

Fig. 4 Cloud chart of road survey points

\section{References}

[1] Li Jian, Li Hong, Liu Tao. Research on the alarm mode of high speed railway obstacle limit violation [J]. Railway communication signal, 2014,50 (11): 67-70.

[2] Xu Chengwei, Zhang Deqiang, Zhang Hao, Wang Baotian, Li Yuetao. Design and implementation of automatic monitoring and alarm system for railway collapse and rockfall based on radar and video analysis technology [J]. Railway computer applications, 2017, 26 (11): 22-27.

[3] Liu Jianbin. Research on monitoring system of railway foreign body intrusion based on Fiber Bragg grating sensor [J]. Transportation science and technology, 2011 (03): 126-128.

[4] Jung J S, Jung C H. Road watch radar system development[C]. Microwave Conference. IEEE, 2014.

[5] Wang Dezhi. Principles of Lidar [J]. Forum for Science and Technology Association (the second half of the month), 2008(05):40.

[6] Chen Pengchao, Ji Junwen, Hou Wei, Lin Xuechun. Design and implementation of an all-solid-state laser monitoring system based on PIC24F microcontroller [J]. Laser Magazine, 2014, 35(04): 33-34+37. 
[7] Zhou Han. Design of cathodic protection current detection system for long-distance pipelines [J]. Electronic Design Engineering, 2019, 27(11): 61-65.

[8] Dou Xinyu, Liang Huaqing. Design of electromagnetic anti-collision signal acquisition system for cluster wells [J]. Instrumentation Technology and Sensors, 2018(02):109-112+138.

[9] Yuan Xia. Three-dimensional laser scanning point cloud data processing and application technology [D]. Nanjing University of Science and Technology, 2006. 